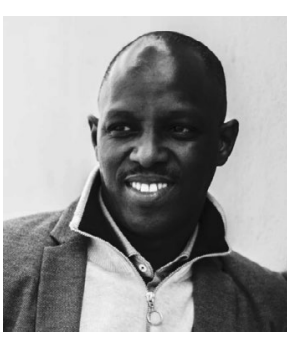

Credit: Marie Laure Marlow

\title{
A genetic research story of giving back and returning to the country of a thousand hills
}

\author{
Would genetics research be a priority for Rwanda while the country was rebuilding just after \\ the 1994 genocide against Tutsi? This was a question that I needed to consider. Sometimes, \\ it is very hard to make the best choice for your career in a new scientific discipline when \\ you have no role models and the only way forward is to start from scratch. Later, however, you \\ can look back on what you have accomplished with surprise, and pride, when you see all \\ your efforts paying off. Here I tell the story of my journey in genetics research, from rebuilding \\ a country after trauma to facing our current COVID-19 pandemic challenges.
}

hav have always been a man with curiosity and ambitions, and growing up in this country of a thousand hills, Rwanda, I loved to be challenged by science. Along my path, a series of remarkable childhood experiences encouraged me to go where my curiosity carried me.

My journey as a scientist started young. When I was growing up, I embarked on various innovative endeavors, such as initiating a project of raising rabbits at my family home and exploring the effects of inbreeding in these rabbits. Through observing some animals with severe malformations that resulted from my rabbit-farming activity, I was unconsciously exposed to the effect of consanguinity in animal evolution, without the underlying knowledge. I was interested in faster growing reproduction to increase productivity, but my lack of scientific knowledge about genetics did not help me think about the law of segregation or use this principle to prevent such types of malformations. Subsequently, I learned about consanguinity theory while pursuing medical studies at the University of Rwanda, and this motivated me later to continue my scientific journey into molecular biology and human genetics to achieve a better understanding these fascinating phenomena.

During this time, there were many challenges faced by every Rwandan after the genocide against the Tutsi in 1994. Despite this, I never lost my courage and commitment to continue shaping my career. After finishing my medical studies at the University of Rwanda, where I got my MD degree in 2003, I decided to pursue a $\mathrm{PhD}$ in human genetics in order to build upon the basic genetic knowledge that had been taught to me, as an undergraduate student at the University of Rwanda, by the late Professor Lucien Koulischer, a recognized leader in human genetics. For my doctoral studies I worked at the Center for Human Genetics at the University of Liège in Belgium headed by Professor Vincent Bours, who, among the many scientists who inspired me, was an early standout. I spent several years working on causal mutations of cystic fibrosis in African populations, where the disease has long been underdiagnosed. This work was the first to identify causal mutations in the genes encoding cystic fibrosis transmembrane conductance regulator (CFTR) and amiloride sensitive epithelial sodium channel $(\mathrm{ENaC})$ in African patients affected by cystic-fibrosis-like disease $^{1}$. I earned my PhD in 2009 and then worked as a postdoctoral researcher studying the pathophysiological role and functional effects of causal mutations in $\mathrm{ENaC}$-encoding genes related to cystic-fibrosis-like disease.

After completing my postgraduate studies, I decided to return to my home country so that I could contribute to helping rebuild Rwanda through my knowledge and efforts. Since then, I have initiated many programs that have positively affected not only the country's economy but also the health of the Rwandan population. Notably, my motivation to come back home was strongly supported by the country's leadership and vision, which encouraged the Rwandan diaspora to return. The Rwandan head of state, President Paul Kagamé, continuously organized outreach programs known as 'Rwanda Day' in which members of the diaspora were called upon to provide their expertise to help rebuild their country.

As I built my career as a medical doctor, I never abandoned my intense curiosity about how things work in the living world. It was not an easy task to start a human genetics program from scratch when national priorities were focused only on rebuilding the post-genocide country and fighting against diseases with major public health effects, such malaria, HIV-AIDS and tuberculosis. This led me to start a journey toward founding the first academic center for human genetics in Rwanda, in order to build molecular technologies and human capacity in genetics and genomics.

Throughout my career, I have been closely involved in clinical services, academics, research, capacity building and management in the health sector. I first served as Director of the Department of Clinical Biology at the Kigali University teaching hospital, which is the largest referral hospital in Rwanda. Later, I was appointed Division Manager of Medical Research at the Rwanda Biomedical Centre, where I coordinated health-related research activities in all national medical institutions to build human capacity to conduct research.

During my career in genetic research, I pioneered new developments and implementations of clinical genetic diagnostic and counseling. I advocated for health insurance coverage for genetic examination of patients in Rwanda, and currently most health insurance covers karyotyping to identify chromosomal abnormalities through cytogenetic and molecular tests. With weekly consultations, parents can now access clinical genetic services offered at the main university teaching hospitals in Rwanda. In the past ten years, since we started offering genetic diagnosis, we have seen improvement in the lives of people with Down's syndrome, or trisomy 21, one of the most common 
chromosomal abnormalities. Upon the birth of a child with suspected Down's syndrome, a multidisciplinary medical plan including systematic cardiac echography check-up, karyotyping and clinical psychological support is offered to the parents. Owing to the increasing awareness of the disease, I and my colleagues have supported parents in creating the Rwanda Down Syndrome Organization (https://rdso.org.rw/wp/), which today follows over 1,000 people with Down's syndrome in its database. The organization is dedicated to improving the lives of people with Down's syndrome through medical care assistance, education and advocacy.

Over the past decade, I have participated in several international research and training collaborations aimed at advancing human genetics and genomics in Africa. Developing such services in Africa presents big challenges due to the lack of adequate infrastructure and financial support. The US National Institutes of Health (NIH) Human Heredity and Health in Africa (H3Africa) program came at the right time to facilitate fundamental research into diseases on the African continent, while also developing infrastructure, resources, training and ethical guidelines to support a sustainable African research enterprise ${ }^{2}$. Our current H3Africa research grant, awarded by the National Institute of Mental Health of the NIH, aims to characterize the transgenerational transmission of the epigenomic impact of genocide exposure and post-traumatic stress disorder (PTSD) in female survivors of the Rwandan genocide and their offspring. Between April and June 1994, almost one million people died in the Rwandan genocide against ethnic Tutsi. Twenty-seven years later, the long-term impact is illustrated by the prevalence of PTSD in Rwandan adults $(26.1 \%)^{5}$, with strong evidence pointing toward the transgenerational transmission of PTSD. PTSD has a known etiologic component: exposure to an extreme traumatic stressor involving a threat to life, which induces a biological cascade that can include epigenetic modification of loci whose activity contributes to psychopathological development ${ }^{3}$. Our pilot research study in Rwanda has shown that transmission of PTSD to the offspring of genocide-exposed mothers is associated with transmission of biological alterations of the hypothalamicpituitary-adrenal (HPA) axis and epigenetic modifications of the gene encoding the glucocorticoid receptor (NR3C1), a key player in the glucocorticoid receptor regulatory network $(\mathrm{GRRN})^{4}$. Building upon these findings, our current work uses genome-scale technologies and focuses initial analyses on the regulation of GRRN and related HPA axis genes, which have been associated with PTSD in epigenome-wide association studies (EWAS) ${ }^{6}$.

During the current COVID-9 pandemic, I learned that my efforts and commitment in returning to my home country constituted the right decision. After observing the role of my research laboratory in the response to COVID-19, particularly in terms of expertise and testing capabilities, I realized that my knowledge of genetics and genomics could now be put to use in supporting the country's efforts in controlling the pandemic.

On 14 March 2020, Rwanda reported its first confirmed case of COVID-19: an asymptomatic traveler arrived in Kigali on 8 March 2020, who presented with symptoms on 13 March 2020 and tested positive the following day. Like most of the world, Rwanda braced for the challenge ahead, expecting that without implementing intensive public health measures, it would experience a rapidly spreading epidemic and subsequent deaths. Many factors predicted that Rwanda, along with other African countries, would experience an even more devastating epidemic than the Europe and the United States due to the weak health systems, incidence of comorbidities, and overcrowded housing conditions. However, through a variety of home-grown solutions and technologies, along with the commitment of Rwandan scientists and government leadership, the COVID-19 pandemic has been contained, and Rwanda has turned out to be one of the countries that responded effectively to the pandemic. Together with my research group at the Centre for Human Genetics at the University of Rwanda, and in collaboration with the National Reference Laboratory (NRL) at the Rwanda Biomedical Centre, we established extensive testing strategies for suspected cases and contacts of cases, as well as through random community sampling using an RT-PCR testing algorithm, which was initiated in March 2020. SARS-CoV-2 infection surveillance was initially centralized at the NRL, which tested 1,000-1,500 samples on a daily basis. Since then, testing capacity has been decentralized and increased to up to 10,000 samples a day - expanding first to four main public laboratories, three in Kigali and one in the Southern Province, and then to seven additional sites in the Northern and Eastern Provinces.

Notwithstanding the establishment of these RT-PCR platforms and capabilities across the country, testing capacity remained a challenge during the pandemic due to the scarcity of resources. Therefore, together with my research group and in collaboration with a scientific research team from the African Institute of Mathematical Sciences (AIMS) led by Professor Wilfred Ndifon, we explored the option of establishing a mass testing technology known as 'pooling testing for SARS-CoV-2' in Rwanda 7 . Based on a hypercube mathematical model developed by AIMS ${ }^{7}$, we validated a strategy of grouping samples before testing them. We used the mathematical algorithm to refine this pooled approach, which analyzes pools of samples from groups of tested people, and then tests a person individually only if a certain pool comes back positive for SARS-CoV-2. The pooled testing approach is most efficient in settings where viral prevalence is relatively low, and this has significantly reduced the cost of RT-PCR testing in Rwanda. The RT-PCR test for SARS-CoV-2 detection costs around US $\$ 50$ per sample, and by pooling, for example, 20 samples together, we saved a lot of resources. This strategy has been scientifically approved to be cost effective during national mass testing, which is exceptionally important in the countries with limited resources.

In addition, the pooled testing strategy has allowed us to decrease the turnaround time for result delivery from 48 hours to 8 hours on average. So far, the method has been scaled up across the country for mass screening in the community, at the airport for arriving passengers and for mass gathering events. Today, this pooling technology has significantly transformed the surveillance of the COVID-19 pandemic in our country as well as across the African continent, where resources are scarce.

In line with my curiosity and drive to implement my own solutions, I have also pioneered a genomics program in the country together with collaborators. We initiated a local genomic sequencing platform for SARS-CoV-2 as a sustainable way to track the dynamics of transmission based on genomic and bioinformatic analysis to identify emerging variants ${ }^{8}$.

Finally, when reflecting on my career journey, as someone who was inspired by great mentors, I appreciate that role models are crucial in science and mentorship. This has prompted me to support young fellows from undergraduate to postgraduate programs. Currently, with my $>40 \mathrm{PhD}$ students working on various research projects, including genetics and genomics, epigenetics, HIV, malaria, hearing loss, cardiovascular diseases, forensic science, cancer, neglected tropical diseases and COVID-19, among others, I still believe that African scientists trained abroad should 
inspire young scientists in Africa to follow their dreams while always thinking about their homes.

Léon Mutesa ${ }^{1,2}$ 网

${ }^{1}$ Center for Human Genetics, College of Medicine and Health Sciences, University of Rwanda, Kigali, Rwanda. ${ }^{2}$ Rwanda National Joint Task Force
COVID-19, Ministry of Health, Kigali, Rwanda.

$凶_{e-m a i l: l m u t e s a @ g m a i l . c o m}$

Published online: 3 March 2022

https://doi.org/10.1038/s41588-022-01028-9

References

1. Mutesa, L. et al. Chest 135, 1233-1242 (2009).
2. H3Africa https://h3africa org (accessed 14 November 2021).

3. Pitman, K. R. et al. Nat. Rev. Neurosci. 13, 769-787 (2012).

4. Perroud, N. et al. World J. Biol. Psychiatry. 15, 334-345 (2014).

5. Mutuyimana, C. et al. BMC Psychol. 7, 84 (2019).

6. Musanabaganwa, C. et al. Epigenomics 14, 11-25 (2022).

7. Mutesa, L. et al. Nature 589, 276-280 (2021).

8. Butera, Y. Nat. Comm. 12, 5705 (2021)

Competing interests

The author declares no competing interests. 